\title{
EVALUACIÓN DE LAS PRUEBAS DOT BLOT Y AGLUTINACIÓN DE LÁTEX PARA EL DIAGNÓSTICO DE CISTICERCOSIS EN PERÚ
}

\author{
Eduardo Miranda-Ulloa ${ }^{1, a,}$ Roxana Sandoval-Ahumada ${ }^{2, b}$, Eduardo Ayala ${ }^{1, c,}$ \\ Juan Vásquez-Ampuero ${ }^{3, d}$
}

\begin{abstract}
RESUMEN
Con el objetivo de evaluar las pruebas dot blot y aglutinación de látex para la detección de cisticercosis humana con antígeno de líquido de cisticerco de Taenia solium, se usaron 125 sueros humanos, de los cuales 60 procedían de personas con cisticercosis confirmada por Western Blot, 45 de personas con otras enfermedades parasitarias y 20 de personas aparentemente sanas. La concentración óptima del antígeno para impregnar las tiras dot blot fue de 0,01 ug/uL, y para impregnar las partículas de látex fue de 0,092 ug/uL. Para la prueba dot blot se encontró una sensibilidad del 100\% y especificidad del 87,7\%; para la aglutinación de látex una sensibilidad del 93,3\% y especificidad del $89,2 \%$. Ambas pruebas podrían ser de utilidad y factibles de implementar como alternativas de diagnóstico serológico en laboratorios de áreas endémicas del Perú.
\end{abstract}

Palabras clave: Cisticercosis; Ensayo de inmunoadsorción enzimática; Pruebas de fijación de látex; Sensibilidad y especificidad (fuente: DeCS BIREME).

\section{ASSESSMENT OF DOT BLOT TESTS AND LATEX AGGLUTINATION FOR CYSTICERCOSIS DIAGNOSTIC IN PERU}

\begin{abstract}
In order to evaluate dot blot tests and latex agglutination for the detection of human cysticercosis with liquid antigen of Taenia solium cysticerci, 125 human sera were used, of which 60 were from people with cysticercosis confirmed by Western Blot, 45 with other parasitic diseases and 20 apparently healthy. The optimal concentration of antigen to impregnate dot blot strips was $0.01 \mathrm{ug} / \mathrm{uL}$, and to impregnate the latex particles was $0.092 \mathrm{ug} / \mathrm{uL}$. For the dot blot test, a sensitivity of $100 \%$ and specificity of $87.7 \%$ was found. For latex agglutination, a sensitivity of $93.3 \%$ and specificity of $89.2 \%$ was found. Both tests may be useful and feasible to implement alternatives of serological diagnosis in laboratories in endemic areas of Peru.
\end{abstract}

Key words: Cysticercosis; Enzyme-linked immunosorbent assay; Latex fixation tests; Sensitivity and specificity (source: MeSH NLM).

\section{INTRODUCCIÓN}

En el diagnóstico de la cisticercosis cerebral se usan análisis serológicos y de imágenes. Los análisis de imágenes como la resonancia magnética (RM) y la tomografía axial computarizada (TAC) son poco accesibles para la población rural, no solo por el costo de la prueba, sino también por la no disponibilidad de los equipos. Los análisis serológicos son útiles para la detección de anticuerpos contra el cisticerco de Taenia solium. La prueba de ELISA se usa como prueba de tamizaje ${ }^{(1,2)}$ y el Western Blot como prueba de confirmación. En el Perú se estandarizó la prueba de Western Blot con antígenos de fluido vesicular de larva de $T$. solium, con una sensibilidad de $91 \%$ y una especificidad del $100 \%$. Sin embargo, esta técnica tiene la desventaja de requerir costosos equipos, que son difíciles de adaptar en el área rural, o por la existencia de laboratorios con infraestructura insuficiente.

\footnotetext{
Centro Nacional de Salud Pública, Instituto Nacional de Salud. Lima, Perú.

Hospital Nacional Hipólito Unanue. Lima, Perú.

Facultad de Medicina Veterinaria, Universidad Nacional Hermilio Valdizán. Huánuco, Perú.

Biólogo, magíster en Microbiología; ${ }^{\mathrm{b}}$ médico patólogo; ${ }^{\mathrm{b}}$ biólogo; ${ }^{\mathrm{d}}$ médico veterinario, magíster en Salud Pública.

Recibido: 11-04-14 Aprobado: 11-06-14
} 
La cisticercosis es endémica en la mayoría de los departamentos del Perú ${ }^{(3,4)}$, sin embargo, aun cuando todos los departamentos cuentan con un Laboratorio de Referencia Regional (LRR) en salud pública, solo Junín, Ayacucho y Cajamarca han implementado un método de tamizaje para la detección de cisticercosis, siendo la mayor dificultad de los LRR para realizar el tamizaje de cisticercosis, la carencia de un lector de ELISA (2).

Las pruebas dot blot y aglutinación de látex fueron usadas como alternativas de diagnóstico para la detección de otras enfermedades. La prueba de látex consiste en una suspensión de partículas de poliestireno sobre las cuales se adhiere el antígeno del parásito. Si esta suspensión se enfrenta a una muestra en la cual hay anticuerpos contra el antígeno, se produce un entrecruzamiento que se visualiza como una aglutinación ${ }^{(5)}$. La prueba de látex ha demostrado buen rendimiento diagnóstico cuando ha sido utilizada para la detección de equinococosis quística, hallando una sensibilidad del $98 \%$ y especificidad del $90 \%{ }^{(6)}$. Existen reportes preliminares acerca de la evaluación de la prueba de aglutinación de látex para la detección de neurocisticercosis con resultados alentadores ${ }^{(7)}$.

La prueba dot blot es denominada ELISA de punto y fue desarrollada como una modificación del ELISA. Es una técnica para la detección de anticuerpos similar al Western Blot, pero difiere en que las proteínas antigénicas no están separadas electroforéticamente, sino que son impregnadas a través de plantillas circulares directamente sobre el papel de nitrocelulosa ${ }^{(8)}$. Un estudio realizado en Colombia concluye que el dot blot es una herramienta diagnóstica útil, sencilla y promisoria para la realización de estudios de cisticercosis en áreas endémicas. Para la ejecución de los ensayos no se requiere de equipos especiales y puede ser utilizado como un ensayo cualitativo para cribar un gran número de muestras ${ }^{(9)}$.

Ante la necesidad de los LRR y laboratorios de hospitales del Ministerio de Salud de diferentes zonas endémicas de cisticercosis, de contar con pruebas de tamizaje más accesibles, el presente estudio tuvo como objetivo evaluar la prueba dot blot y aglutinación de látex para la detección de cisticercosis en el Perú.

\section{EL ESTUDIO}

Estudio transversal de evaluación de prueba diagnóstica realizado durante los años 2011-2012. Las muestras fueron seleccionadas de la seroteca del Laboratorio de Referencia Nacional de Zoonosis Parasitaria del Instituto Nacional de Salud del Perú. El estudio tuvo cuatro etapas diferenciadas: selección del panel de sueros positivos y negativos a cisticercosis; preparación del antígeno de cisticerco; preparación del dot blot y aglutinación de látex, y determinación del rendimiento diagnóstico de las dos pruebas. A continuación se describe cada una de las etapas.

\section{SELECCIÓN DEL PANEL DE SUEROS REFERENTES}

Se seleccionó por conveniencia 125 sueros, de los cuales, 60 procedieron de pacientes con cisticercosis confirmada mediante Western Blot, que constituyo el gold standard; 20 sueros fueron de personas aparentemente sanas, y 45 sueros procedieron de pacientes con otras enfermedades: giardiasis (7), hymenolepiosis (6), ascariosis (6), entamoebiosis (5), Endolimax nana (4), hidatidosis (4), fasciolosis (4), toxoplasmosis (3), trichuriosis (2), blastocystosis (2) y strongyloidiosis (2).

A los sueros procedentes de personas aparentemente sanas y con otras enfermedades, se les confirmó su negatividad a cisticercosis mediante la prueba de Western Blot. Se define como persona aparentemente sana aquella que luego de realizarle un chequeo médico general clínico y laboratorial, presenta resultados negativos a todos sus análisis.

\section{PREPARACIÓN DEL ANTÍGENO DE CISTICERCO}

Para la obtención de líquido de cisticerco de $T$. solium se seleccionó un cerdo naturalmente infectado con cisticercos de $T$. solium de una zona endémica. El líquido fue extraído y centrifugado a $40000 \mathrm{rpm}$ durante una hora a $4{ }^{\circ} \mathrm{C}$. El sedimento fue descartado. En el sobrenadante se determinó la concentración de proteínas por el método de Lowry. La concentración del antígeno fue de 1,2 ug/uL. La caracterización del antígeno de cisticerco se realizó mediante electroforesis en gel de poliacrilamida para confirmar y caracterizar los componentes proteicos ${ }^{(10)}$.

\section{PREPARACIÓN DE LA TÉCNICA DOT BLOT Y AGLU- TINACIÓN DE LÁTEX}

\section{TÉCNICA DOT BLOT}

Impregnación del antígeno. Se impregnó cada tira de nitrocelulosa (marca biorad) de $4 \times 20 \mathrm{~mm}$ con $5 \mathrm{uL}$ de antígeno de cisticerco a una concentración óptima de $0,01 \mathrm{ug} / \mathrm{uL}$.

Método de ensayo. Cada tira fue saturada durante 30 min con PBS- Tween-0,05\%, leche descremada al 5\% (PBS-TL). La determinación de anticuerpos se realizó mediante incubación de $10 \mathrm{uL}$ de suero diluido en $1 \mathrm{~mL}$ de PBS-TL por una hora a temperatura entre 18 a $28^{\circ} \mathrm{C}$. 
Se lavó con $1 \mathrm{~mL}$ de PBS- Tween-0,05\% (PBS-T) por 5 $\mathrm{min}$, por cinco veces. Se adicionó un conjugado anti-lgG humano marcado con peroxidasa ( $1 \mathrm{uL}$ diluido en $1 \mathrm{~mL}$ de de PBS-TL) por una hora. Luego se lavó las tiras con $1 \mathrm{~mL}$ de PBS T durante $5 \mathrm{~min}$, por tres veces, y dos veces con solución PBS pH 7,2. Se reveló la reacción por ocho minutos agregando $1 \mathrm{~mL}$ de PBS, $0,5 \mathrm{mg}$ de diaminobencidina más $1 \mathrm{uL}$ de $\mathrm{H}_{2} \mathrm{O}_{2}$. Finalmente, se lavó con agua destilada por 8 a 10 veces y se dejó secar. Todo el procedimiento se realizó a temperatura entre 18 a $28^{\circ} \mathrm{C}$ y en agitación constante.

Interpretación de resultados. Consistió en visualizar la presencia de un punto de color marrón (reactivo o positivo) o la ausencia de este (no reactivo o negativo). Cada ensayo fue considerado válido si los controles positivo y negativo reaccionaron concordantemente.

\section{TÉCNICA AGLUTINACIÓN DE LÁTEX}

Impregnación del antígeno de cisticerco a las partículas látex. Se empleó partículas látex de poliestireno de $0,25 \mu \mathrm{m}$ procedentes de Bangs Laboratories EE. UU., la suspensión stock se preparó diluyendo la suspensión comercial $1: 8$ en buffer glicina $\mathrm{pH}: 8,2$. $(9 \mathrm{~g} \mathrm{NaCl}, 1 \mathrm{~g}$ de $\mathrm{CaCl}_{2}, 7,31 \mathrm{~g}$ de glicina, $0,10 \mathrm{~g}$ de thimerosal y un litro de agua destilada). La concentración del antígeno para la impregnación de las partículas fue de 0,092 ug/uL. (Reactivo de látex-cisticercosis).

Método de ensayo. La prueba se realizó en una placa de plástico fondo oscuro. Se colocó $20 \mu \mathrm{L}$ del suero más $20 \mu \mathrm{L}$ del reactivo de látex-cisticercosis y se homogeneizó en un agitador de placas durante $30 \mathrm{~min}$.

Interpretación de resultado. La aglutinación de las partículas de látex correspondió a un resultado positivo. En los sueros negativos las partículas permanecieron en suspensión. Cada ensayo fue considerado válido si los controles positivo y negativo reaccionaron concordantemente.

El análisis estadístico de la determinación del rendimiento diagnóstico de las dos pruebas se realizó mediante la sensibilidad y especificidad ${ }^{(11)}$.

El protocolo del estudio fue aprobado por el comité de investigación y el comité de ética del Instituto Nacional de Salud del Perú.

\section{HALLAZGOS}

Las proteínas del antígeno de cisticerco de $T$. solium visualizados en geles de poliacrilamida, fueron catorce: fracciones $13,14,17,18,23,24,31,35,42,50,66$,
Tabla 1. Resultados de las pruebas dot bloty aglutinación de látex usando sueros de pacientes con cisticercosis, con otras parasitosis y personas aparentemente sanas

\begin{tabular}{|c|c|c|c|c|}
\hline \multirow{2}{*}{ Enfermedad } & \multicolumn{2}{|c|}{ Dot blot } & \multicolumn{2}{|c|}{$\begin{array}{c}\text { Aglutinación } \\
\text { de látex }\end{array}$} \\
\hline & Positivos & Negativos & Positivos & Negativos \\
\hline Cisticercosis & 60 & 0 & 56 & 4 \\
\hline Entamoebiosis & 0 & 5 & 0 & 5 \\
\hline Hymenolepiosis & 0 & 6 & 0 & 6 \\
\hline Ascariosis & 1 & 5 & 0 & 6 \\
\hline Trichuriosis & 0 & 2 & 0 & 2 \\
\hline Giardiasis & 2 & 5 & 2 & 5 \\
\hline Strongioloidiosis & 1 & 1 & 1 & 1 \\
\hline Endolimax nana & 0 & 4 & 0 & 4 \\
\hline Blastocystosis & 0 & 2 & 0 & 2 \\
\hline Hidatidosis & 2 & 2 & 2 & 2 \\
\hline Toxoplasmosis & 1 & 2 & 0 & 3 \\
\hline Fasciolosis & 1 & 3 & 2 & 2 \\
\hline $\begin{array}{l}\text { Aparentemente } \\
\text { sanos }\end{array}$ & 0 & 20 & 0 & 20 \\
\hline
\end{tabular}

97, 148 y $250 \mathrm{KDa}$. En este grupo se confirmó la presencia de ocho proteínas diagnósticas específicas a cisticercosis, estas fracciones fueron: 13, 14, 17, 18, 23, 24,31 y $35 \mathrm{Kda}$.

En la prueba dot blot no se encontró ningún suero falso negativo con la concentración de 0,01 ug/uL de antígeno utilizado en la preparación de las tiras. No se encontró reacción cruzada con sueros de personas aparentemente sanas, ni sueros de pacientes con entamoebiosis, hymenolepiosis, trichuriosis, Endolimax nana y blastocystosis (Tabla 1). El ensayo duró $3 \mathrm{~h} 30 \mathrm{~min}$. La sensibilidad fue de $100 \%$ y la especificidad de $87,7 \%$.

En la prueba de látex se encontró que la concentración óptima deł antígeno para impregnar las partículas fue de 0,092 ug/uL. No se reportó reacción cruzada con sueros de personas aparentemente sanas, ni con sueros de pacientes con entamoebiosis, hymenolepiosis, ascariosis, trichuriosis, Endolimax nana, blastocystosis y toxoplasmosis (Tabla 1). El ensayo duró $30 \mathrm{~min}$. La sensibilidad fue de $93,3 \%$ y la especificidad de $89,2 \%$.

\section{DISCUSIÓN}

Los hallazgos mostraron mejor rendimiento del antígeno de líquido de cisticerco con muestras de suero, lo cual resulto superior al uso de antígenos purificados y antígenos de membrana de cisticerco con muestras de LCR. La mayoría de los antígenos purificados proceden del líquido de cisticerco debido a que constituye la mayor fuente de antígenos del cisticerco de $T$. solium ${ }^{(12,13)}$. Otros reportes relacionados al diagnóstico 
de cisticercosis señalaron al dot blot como una prueba con buen rendimiento diagnóstico para la detección de anticuerpos contra cisticercos ${ }^{(14)}$. En este trabajo no se encontró reacciones cruzadas con sueros de personas aparentemente sanas ni con sueros de pacientes con otras enfermedades parasitarias, lo cual indica que no hubo inmunógenos comunes de esas parasitosis con el antígeno de cisticerco preparado.

Un estudio de evaluación de dot blot para cisticercosis reportó una sensibilidad de $91,1 \%$, y una especificidad de $100 \%$ usando 125 sueros y siete glicoproteínas: (GP 50, GP 42-39, GP 24, GP 21, GP 18, GP 14 Y GP 13) ${ }^{(13)}$. Al contrastar con los resultados de la presente investigación, la sensibilidad que se obtuvo alcanzó el $100 \%$, sin embargo, la especificidad alcanzada fue menor. Las diferencias entre ambos estudios se puede atribuir al tipo de antígeno usado; ya que el uso de antígenos purificados incrementa la especificidad de la prueba ${ }^{(14)}$.

En otro estudio sobre dot blot usando antígeno purificado GP 24 contra 44 sueros de pacientes negativos a cisticercosis, pero con otras enfermedades parasitarias, se reportó $100 \%$ de especificidad. Antígenos de extractos de membrana y líquido de cisticerco evaluados contra un mismo panel de muestras de LCR, reportaron una sensibilidad de 95,1 a $97,6 \%$ y una especificidad de 96,9 y $100 \%$ respectivamente ${ }^{(15)}$.

La prueba de dot blot presentó reacción cruzada con sueros de pacientes con ascariosis, giardiosis, estrongiloidiosis, hidatidosis, toxoplasmosis y con fasciolosis. No obstante, dado el pequeño número de muestras con estas parasitosis no es posible afirmar que haya reacciones cruzadas importantes entre la prueba de este trabajo y estas parasitosis. Por otra parte, es relevante señalar que la mayoría de los pacientes con estas enfermedades resultaron negativos a cisticercosis.

Sobre el uso de la prueba de aglutinación de látex para cisticercosis, sólo se encontró dos reportes previos luego de buscar en publicaciones nacionales e internacionales. Un estudio realizado en Brasil evaluó la prueba de látex usando 43 muestras de LCR, y obtuvo una sensibilidad de $89,5 \%$ y una especificidad de $75 \%{ }^{(5)}$ La razón de tener menor sensibilidad y especificidad que la obtenida en el presente estudio, sería el tipo de muestra evaluada; está descrito que los anticuerpos contra cisticerco de $T$. solium primero aparecen en suero y luego en LCR en pacientes con neurocisticercosis ${ }^{(3)}$.

La prueba de látex también fue evaluada para la detección de antígeno de cisticerco de T. solium en LCR y en suero, reportándose resultados de sensibilidad de 64,7 y $52,1 \%$ y especificidad de 85,7 y $96 \%$ respectivamente. A pesar que la sensibilidad fue baja, esta prueba es relevante por su buena especificidad, ya que el test es usado para el seguimiento de los pacientes con neurocisticercosis luego de su terapia ${ }^{(8)}$. La aparición de anticuerpos en suero depende de una mínima cantidad de inmunógenos. El hecho de que la sensibilidad no alcance el $100 \%$, podría atribuirse a que algunos pacientes con un solo cisticerco no produzcan la suficiente cantidad de anticuerpos para ser detectada por alguna prueba en particular.

La prueba de látex presentó reacción cruzada con sueros de pacientes con giardiosis, estrongiloidiosis, hidatidosis, y fasciolosis, la posible razón para estas reacciones sería el uso de un antígeno que no solo tiene proteínas específicas, sino también inespecíficas, las cuales son comunes entre parásitos. Actualmente, esta prueba se usa de manera habitual en los LRR del Perú y solo las muestras positivas pasan a ser confirmadas por Inmunoblot.

Recomendamos procesar la prueba de látex en ambientes que no tengan demasiada ventilación, con la finalidad de evitar que durante el ensayo las muestras y el reactivo se sequen. A diferencia de otras técnicas, la prueba de látex es rápida, más sencilla y no requiere de sofisticados equipos para su desarrollo.

Entre las limitaciones del presente estudio se puede mencionar que no fue posible conocer si los pacientes con cisticercosis presentaron cisticercosis muscular o subcutánea. La continuación del estudio involucra evaluar estas pruebas en campo, midiendo la estabilidad y el tiempo de expiración de los reactivos para determinar la reproducibilidad del kit y sus valores predictivos positivos y negativos.

Se concluye que ambas pruebas podrían ser de utilidad como tamizaje en estudios de rutina en pacientes con sospecha de cisticercosis y en investigaciones epidemiológicas en campo. Se espera que en un futuro el Ministerio de Salud a través de la Estrategia Sanitaria Nacional de Zoonosis y el Instituto Nacional de Salud del Perú puedan producir estos dos kits in house y lo distribuyan a los laboratorios regionales en áreas rurales y endémicas del Perú, para de esta manera contribuir al control y vigilancia epidemiológica de la cisticercosis humana.

Contribuciones de autoría: EMU y RSA participaron en la concepción y diseño del artículo, recolección de resultados, análisis e interpretación de datos, redacción del artículo, revisión crítica del artículo. EA y JVA participaron en la concepción y diseño del artículo, aporte de material de estudio, revisión crítica del artículo.

Fuentes de financiamiento: Instituto Nacional de Salud.

Conflictos de interés: los autores declaran no tener conflictos de interés. 


\section{REFERENCIAS BIBLIOGRÁFICAS}

1. Instituto Nacional de Salud. Manual de Procedimientos para el diagnóstico serológico de las zoonosis parasitarias. Serie de Normas Técnicas No 32. 2da ed. Lima: INS; 2010.

2. Escalante H, Miranda E, Lorca M, Verastegui M, Torres R. La técnica de "Western Blot" con antígenos de fluido vesicular de Cysticercus cellulosae para el diagnóstico de la cisticercosis. Bol Per Parasitol. 1995;11(1):26-31.

3. García HH, Gonzales A, Martínez SM, Gilman RH. Teniasis/cisticercosis por Taenia solium, un serio problema de salud pública en el Perú. Lima: Ministerio de Salud del Perú; 2001.

4. Cordero A, Miranda E, Segovia G, Cantoral V, Huarcaya I. Prevalencia de teniosis y seroprevalencia de cisticercosis humana en Pampa Cangallo, Ayacucho, Perú, 2008. Rev Peru Med Exp Salud Publica. 2010;27(4):562-8.

5. Rocha SM, Suzuki LA, Silva AD, Arruda GC, Rossi CL. A rapid latex agglutination test for the detection of anti-cysticercus antibodies in cerebrospinal fluid (CSF). Rev Inst Med Trop Sao Paulo. 2002 JanFeb;44(1):57-8.

6. Miranda E, Sánchez E, Náquira C, Somocurcio J, Ayala E, Miranda
G. Evaluación de una prueba de aglutinación de látex para el diagnóstico serológico de la equinococosis quística. Rev Peru Med Exp Salud Publica. 2009;26(2):198-202.

7. Biswas R, Parija SC. A rapid slide agglutination test for the diagnosis of neurocysticercosis in the rural health set up. Trop Parasitol. 2011 Jul;1(2):948. doi: 10.4103/2229-5070.86942.

8. Mondragón A, Plancarte A, Flisser A. El diagnóstico de la cisticercosis humana por ELISA. Salud Publica Mex. 1994;36(4):393-8.

9. Agudelo P, Botero D, Palacio LG. Evaluación del método ELISA de punto para el diagnóstico de la Cisticercosis humana y para estimar valores de prevalencia en una región endémica en Colombia. Biomedica. 2005;25(4):48895.

10. Instituto Nacional de Salud. Manual de procedimientos de electroforesis para proteínas y ADN. Serie de Normas Técnicas No 38. Lima: INS; 2003.

11. Organización Panamericana de la Salud. Manual de procedimientos de control de calidad para los laboratorios de serología de los bancos de sangre. Washington DC: OPS; 1994.
12. Saavedra H, Gonzales I, Alvarado M, Porras M, Vargas M, Cjuno R, et al. Diagnóstico y manejo de la neurocisticercosis en el Perú. Rev Peru Med Exp Salud Publica. 2010;27(4):586-91.

13. Plancarte A, Fexas M, Flisser A. Reactivity in ELISA and dot blot of purified GP24, an immunodominant antigen of Taenia solium, for the diagnosis of human neurocysticercosis. Int J Parasitol. 1994 Aug;24(5):733-8.

14. Agudelo Flórez P, Palacio L. Dot blot para el diagnóstico de la cisticercosis porcina. Rev Sci Tech Off Int Epiz. 2009;28(3):1077-84.

15. Vaz AJ, Nakamura PM, Camargo ME, Camargo ED, Ferreira AW. Dot-ELISA for the detection of anti-Cysticercus cellulosae antibodies in cerebrospinal fluid using a new solid phase (resintreated polyester fabric) and Cysticercus longicollis antigens. Rev Inst Med Trop Sao Paulo. 1996 Nov-Dec;38(6):391-6.

Correspondencia: Eduardo Miranda Ulloa Dirección: Cápac Yupanqui 1400, Lima11, Perú.

Teléfono: 959602884

Correo electrónico:fernandoul@hotmail.com

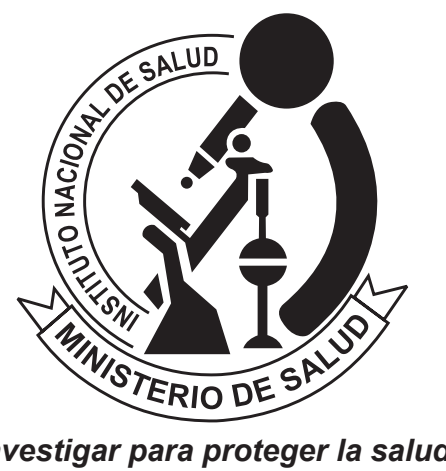

REVISTA PERUANA DE MEDICINA EXPERIMENTAL Y SALUD PÚBLICA CUMPLIENDO SUS METAS Y PROYECTÁNDOSE AL FUTURO

\section{Visite los contenidos de la revista en: www.ins.gob.pe/rpmesp}

